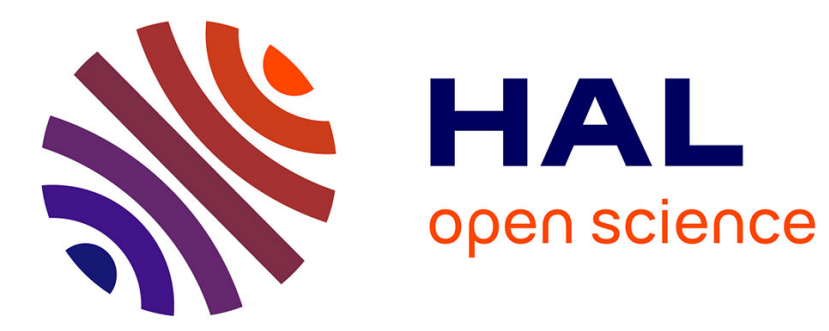

\title{
Riccati observer design for homography decomposition
}

Ninad Manerikar, Minh-Duc Hua, Simone de Marco, Tarek Hamel

\section{To cite this version:}

Ninad Manerikar, Minh-Duc Hua, Simone de Marco, Tarek Hamel. Riccati observer design for homography decomposition. European Control Conference, 2020, Saint Petersburg, Russia. 10.23919/ECC51009.2020.9143740 . hal-03052530

\section{HAL Id: hal-03052530 \\ https://hal.science/hal-03052530}

Submitted on 10 Dec 2020

HAL is a multi-disciplinary open access archive for the deposit and dissemination of scientific research documents, whether they are published or not. The documents may come from teaching and research institutions in France or abroad, or from public or private research centers.
L'archive ouverte pluridisciplinaire HAL, est destinée au dépôt et à la diffusion de documents scientifiques de niveau recherche, publiés ou non, émanant des établissements d'enseignement et de recherche français ou étrangers, des laboratoires publics ou privés. 


\title{
Riccati observer design for homography decomposition
}

\author{
Ninad Manerikar, Minh-Duc Hua, Simone De Marco and Tarek Hamel
}

\begin{abstract}
This paper presents a novel approach for tackling the classical problem of homography decomposition. The novelty of this paper lies in the design of a deterministic Riccati observer for addressing the homography decomposition problem instead of solving it on a frame-by-frame basis like traditional algebraic approaches. By exploiting the temporal correlation of image sequences and the natural low-pass response of the observer, we believe that the resulting estimates are robust and less prone to measurement noise. Moreover, an extensive observability analysis that points out a sufficient uniform observability condition under which local exponential stability is granted has been carried out. The large domain of convergence and good performance of the proposed observer have been demonstrated through simulation results.
\end{abstract}

\section{INTRODUCTION}

When a robotic vehicle equipped with a monocular camera navigates in an environment composed of (near) planar surfaces such as man-made structures or a ground terrain distantly viewed by an unmanned aerial vehicle, then the planar nature of the environment can be exploited in vision processing algorithms as well as vision-based control algorithms. Two different images of the same planar surface are typically related by the so-called homography mapping that essentially encodes the camera's pose (i.e. position and orientation), the distance between the camera and the scene and its normal vector in one single matrix [3]. Homographies have been extensively used in robot control as a vision primitive, and one of the most successful visual servo control paradigms is the $2 \frac{1}{2} \mathrm{D}$ approach proposed by Malis et al. [5] that relies on the extraction of the camera displacement (i.e. orientation and translation up to a scale factor) and the scene's normal vector from homography (assuming that the camera's intrinsic parameters are known). This process of extraction is termed as Euclidean homography decomposition, also called Euclidean reconstruction from homography.

Classical approaches for homography decomposition such as Faugeras SVD-based [1], Zhang SVD-based [9] algorithms use the singular value decomposition to obtain numerical solutions. Malis and Vargas [6] lately solved the homography decomposition problem with an analytical approach, making it more suitable for real-time robot control applications. In that work, not only analytical expressions of the decomposition elements are provided, valuable insights regarding the robustness of vision-based control laws are also analyzed. In summary, to our knowledge all existing homography decomposition methods belong to algebraic category that only focuses on solving the homography decomposition

N. Manerikar, M.-D. Hua, S. De Marco, T. Hamel are with I3S, Université Côte d'Azur, CNRS, Sophia Antipolis, France, manerika(hua, sdemarco, hamel)@i3s.unice.fr. problem on a frame-by-frame basis, but not on filtering measurement noise. The precision of the decomposition elements (particularly of the estimated normal vector) is thus highly prone to noise, especially when the camera's translation is small [6]. The problem even becomes degenerate when the latter vanishes. Robotic vehicle applications, however, provide temporal sequences of images together with inertial measurements (from an embedded Inertial Measurement Unit). Therefore, it seems natural to exploit the temporal correlation rather than try to solve homography decomposition for each pair of image frames.

In this paper, a novel direction for solving the homography decomposition by exploiting the system dynamics is explored. The proposed solution is developed in the form of a nonlinear observer derived from the deterministic Riccati observer design framework recently proposed in [2]. We believe that the resulting estimated quantities would be less noisy since the noise can be filtered through a natural lowpass response of the observer. Moreover, the challenging theoretical issue related to the uniform observability, under which local exponential stability is granted, has been carefully addressed, and simulation results demonstrate a good performance and a large attraction domain of the proposed observer.

\section{THEORETICAL BACKGROUND}

\section{A. Notation}

- $\left\{e_{1}, e_{2}, e_{3}\right\}$ denotes the canonical basis of $\mathbb{R}^{3}$ and the identity matrix of $\mathbb{R}^{n \times n}$ is denoted as $I_{n}$.

- $(\cdot)_{\times}$denotes the skew-symmetric matrix associated with the cross product, i.e. $u_{\times} v=u \times v, \forall u, v \in \mathbb{R}^{3}$.

- $\pi_{u} \triangleq I_{3}-u u^{\top}, \forall u \in S^{2}$, is the projection operator onto the plane orthogonal to $u$.

\section{B. Brief overview of the Riccati observer design framework}

The observer proposed in this paper is based on the Riccati design framework recently developed in [2]. The following nonlinear system (which is a particular case of the class of systems considered in [2]) is investigated:

$$
\left\{\begin{array}{l}
\dot{X}=A(t) X+U+O\left(|X|^{2}\right)+O(|X||U|) \\
Y=C(X, t) X+O\left(|X|^{2}\right)
\end{array}\right.
$$

with state $X=\left[X_{1}^{\top}, X_{2}^{\top}\right]^{\top}, X_{1} \in \mathcal{B}_{r}^{n_{1}}$ (the closed ball in $\mathbb{R}^{n_{1}}$ of radius $r$ ), $X_{2} \in \mathbb{R}^{n_{2}}$, output $Y \in \mathbb{R}^{m}, A(t)$ a continuous matrix-valued function uniformly bounded with respect to (w.r.t.) $t$ in the form

$$
A(t)=\left[\begin{array}{ll}
A_{1,1}(t) & 0_{n_{1} \times n_{2}} \\
A_{2,1}(t) & A_{2,2}(t)
\end{array}\right]
$$


and $C(X, t) \in \mathbb{R}^{m \times\left(n_{1}+n_{2}\right)}$ a continuous matrix-valued function uniformly continuous w.r.t. $X$ and uniformly bounded w.r.t. $t$. Then, apply the input

$$
U=-P C^{\top} D Y
$$

with $P \in \mathbb{R}^{\left(n_{1}+n_{2}\right) \times\left(n_{1}+n_{2}\right)}$ a symmetric positive definite matrix solution to the following continuous Riccati equation (CRE):

$$
\dot{P}=A P+P A^{\top}-P C^{\top} D(t) C P+S(t)
$$

with $P(0) \in \mathbb{R}^{\left(n_{1}+n_{2}\right) \times\left(n_{1}+n_{2}\right)}$ a symmetric positive definite matrix, $D(t) \in \mathbb{R}^{m \times m}$ bounded continuous symmetric positive semi-definite, and $S(t) \in \mathbb{R}^{\left(n_{1}+n_{2}\right) \times\left(n_{1}+n_{2}\right)}$ bounded continuous symmetric positive definite.

Then, from Theorem 3.1 and Corollary 3.2 in [2], $X=0$ is locally exponentially stable (LES) when both matrices $D(t)$ and $S(t)$ are larger than some constant positive matrix and the pair $\left(A^{\star}(t), C^{\star}(t)\right)$, with $A^{\star}(t) \triangleq A(t), C^{\star}(t) \triangleq C(0, t)$, is uniformly observable.

\section{Recalls of uniform observability}

Definition 1 (uniform observability) The pair $(A(t), C(t))$ is called uniformly observable if there exist $\delta, \mu>0$ such that $\forall t \geq 0$

$W(t, t+\delta) \triangleq \frac{1}{\delta} \int_{t}^{t+\delta} \Phi^{\top}(t, \tau) C^{\top}(\tau) C(\tau) \Phi(t, \tau) \mathrm{d} \tau \geq \mu I_{n}$

with $\Phi(t, \tau)$ the transition matrix associated with $A(t)$, i.e. such that $\frac{d}{d t} \Phi(t, \tau)=A(t) \Phi(t, \tau)$ with $\Phi(t, t)=I_{n}$.

$W(t, t+\delta)$ is called the observability Gramian. The following lemma [8] establishes a sufficient condition of uniform observability.

Lemma 1 (see [8]) If there exists a matrix-valued function $M(\cdot)$ of dimension $(p \times n)(p \geq 1)$ composed of row vectors of $N_{0}=C, N_{k}=N_{k-1} A+\dot{N}_{k-1}, k=1, \cdots$ such that for some positive numbers $\bar{\delta}, \bar{\mu}$ and $\forall t \geq 0$

$$
\frac{1}{\bar{\delta}} \int_{t}^{t+\bar{\delta}} \operatorname{det}\left(M^{\top}(\tau) M(\tau)\right) \mathrm{d} \tau \geq \bar{\mu}
$$

then $W(t, t+\delta)$ satisfies condition (5).

\section{Problem statement}

\section{A. Homography decomposition problem}

Consider a moving camera observing a textured planar scene. Let $\{\mathcal{A}\}$ (resp. $\{\mathcal{B}\}$ ) denote the reference (resp. current) right-hand frame attached to the camera at the reference (resp. current) view. Let $\xi \in \mathbb{R}^{3}$ denote the position of frame $\{\mathcal{B}\}$ w.r.t. frame $\{\mathcal{A}\}$ expressed in frame $\{\mathcal{A}\}$. The orientation of frame $\{\mathcal{B}\}$ w.r.t. frame $\{\mathcal{A}\}$ is represented by a rotation matrix $R \in \mathrm{SO}(3)$. The pose of the camera $(R, \xi)$ determines a rigid body transformation from $\{\mathcal{B}\}$ to $\{\mathcal{A}\}$.

The so-called Euclidean homography that maps Euclidean coordinates of the scene's points from $\{\mathcal{B}\}$ to $\{\mathcal{A}\}$ is given by (see Fig. 1)

$$
H=R+\frac{1}{d} \xi \eta^{\top}
$$

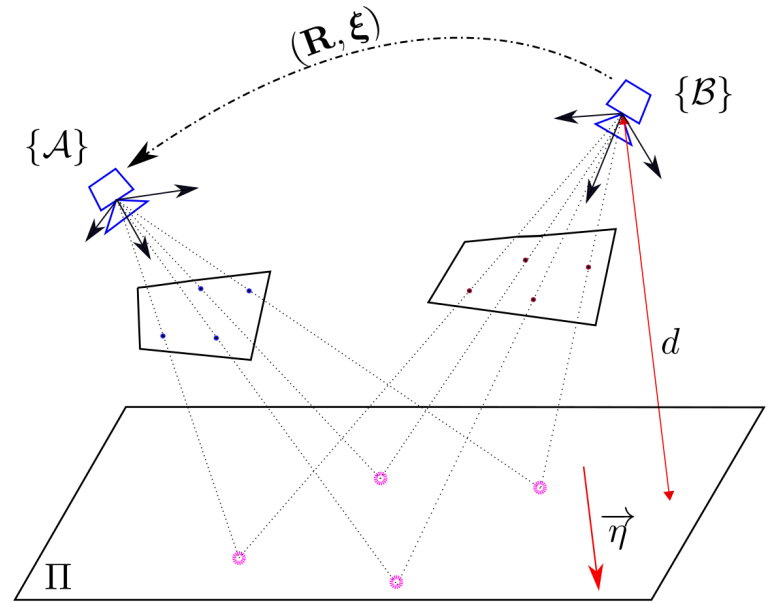

Fig. 1. Euclidean homography relating the camera's pose, the distance to the plane and the plane's normal vector by $H=R+\frac{1}{d} \xi \eta^{\top}$.

where $d$ and $\eta$ are, respectively, the normal distance from the origin of frame $\{\mathcal{B}\}$ to the observed scene and the normal vector pointing towards the scene expressed in $\{\mathcal{B}\}$. Assume that the camera is well calibrated, then the Euclidean homography can be directly computed from the so-called projective homography or image homography estimated from image point correspondences (see [6] for details).

The well-known problem of Euclidean homography decomposition consists in decomposing the matrix $H$ into the elements $R, \frac{\xi}{d}$ and $\eta$. Note that expression (7) of $H$ can be rewritten as

$$
H=R\left(I_{3}+\bar{\xi} \eta^{\top}\right)
$$

with

$$
\bar{\xi}:=\frac{R^{\top} \xi}{d}
$$

Since $\frac{\xi}{d}$ can be computed as $R \bar{\xi}$, decomposing $H$ into $\left(R, \frac{\xi}{d}, \eta\right)$ is equivalent to decomposing it into $(R, \bar{\xi}, \eta)$.

In this paper, we propose to explore a novel direction of solving this homography decomposition problem dynamically by exploiting the differential equations guiding these variables (i.e. $R, \bar{\xi}, \eta$ ). The underlying idea is to design an observer allowing for an exponential convergence rate of the state estimates $(\hat{R}, \hat{\bar{\xi}}, \hat{\eta})$ to the real state variables $(R, \bar{\xi}, \eta)$.

$B$. System equations and measurements for observer design The rigid body kinematics of $(R, \xi)$ are given by

$$
\left\{\begin{array}{c}
\dot{R}=R \Omega_{\times} \\
\dot{\xi}=R V
\end{array}\right.
$$

with $V \in \mathbb{R}^{3}$ and $\Omega \in \mathbb{R}^{3}$ denoting the linear and angular velocities expressed in $\{\mathcal{B}\}$.

Since the scene is stationary the normal vector $i \dot{\eta} \in S^{2}$ expressed in the reference frame $\{\mathcal{A}\}$ is constant. Since $\eta=$ $R^{\top} \stackrel{\eta}{\eta}$ one thus deduces

$$
\dot{\eta}=-\Omega_{\times} \eta
$$

Using (9) and (10), one verifies that

$$
\begin{aligned}
\dot{\bar{\xi}} & =-\Omega_{\times} \bar{\xi}+\frac{V}{d}-\frac{\dot{d}}{\bar{d}} \bar{\xi} \\
& =\left(-\Omega_{\times}+\phi_{\perp} I_{3}\right) \bar{\xi}+\phi
\end{aligned}
$$


where $\phi:=\frac{V}{d}$ and $\phi_{\perp}:=-\frac{\dot{d}}{d}=\frac{V^{\top} \eta}{d}$ are the so-called translational optical flow and optical flow divergence, respectively. Both the translational optical flow and the optical flow divergence are assumed to be measured (see our prior work on how these quantities can be estimated [7]). Assume also that the angular velocity $\Omega$ is also measured using an embedded 3-axes gyrometers.

In this paper we focus on developing a nonlinear observer allowing for dynamically decomposing the homography matrix $H$ into $(R, \bar{\xi}, \eta)$ by exploiting the system dynamics (10)(12), i.e.

$$
\left\{\begin{array}{l}
\dot{\eta}=-\Omega_{\times} \eta \\
\dot{R}=R \Omega_{\times} \\
\dot{\bar{\xi}}=\left(-\Omega_{\times}+\phi_{\perp} I_{3}\right) \bar{\xi}+\phi
\end{array}\right.
$$

and using the homography measurement equation (8) together with the measurements of $\left(\Omega, \phi, \phi_{\perp}\right)$.

\section{OBSERVER DESIGN}

\section{A. Observer derivation}

Inspired by [4], we avoid using minimal parametrization techniques such as spherical coordinate system to parametrize the normal unit vector $\eta-$ an element of $S^{2}$. Instead, an auxiliary rotation matrix $Q \in S O(3)$ is introduced such that

$$
\eta=Q^{\top} e_{3}
$$

The underlying idea is to over-parameterize an element of $S^{2}$ (dimension 2) by an element of $S O(3)$ (dimension 3 ). The advantage of such type of parameterization is that it reduces the complexities of the error system in first order approximations that arise due to minimal parameterization techniques for elements on $S^{2}$ (see [4] for more thorough discussions).

In view of the dynamics (11) of $\eta$, one deduces a possibility of the dynamics of $Q$ as

$$
\dot{Q}=Q \Omega_{\times}
$$

In view of (13) and (14), the following general form of observer is proposed

$$
\left\{\begin{array}{l}
\dot{\hat{Q}}=\hat{Q} \Omega_{\times}-\sigma_{Q \times} \hat{Q} \\
\dot{\hat{R}}=\hat{R} \Omega_{\times}-\hat{R} \sigma_{R \times} \\
\dot{\hat{\bar{\xi}}}=\left(-\Omega_{\times}+\phi_{\perp} I_{3}\right) \hat{\bar{\xi}}+\phi-\sigma_{\bar{\xi}}
\end{array}\right.
$$

with initial conditions $\hat{Q}(0), \hat{R}(0) \in S O(3), \hat{\bar{\xi}}(0) \in \mathbb{R}^{3}$ and with innovation terms $\sigma_{Q}, \sigma_{R}, \sigma_{\bar{\xi}} \in \mathbb{R}^{3}$ to be designed thereafter. The estimated normal vector is then given by

$$
\hat{\eta}:=\hat{Q}^{\top} e_{3}
$$

The following error variables are defined:

$$
\tilde{Q}:=Q \hat{Q}^{\top}, \tilde{R}:=\hat{R}^{\top} R, \tilde{\bar{\xi}}:=\bar{\xi}-\hat{\bar{\xi}}
$$

Then the objective of observer design consists in stabilizing $\left(\tilde{Q} e_{3}, \tilde{R}, \tilde{\xi}\right)$ about $\left(e_{3}, I_{3}, 0\right)$.
From (10), (15) and (16), one verifies that the error system is given by

$$
\left\{\begin{array}{l}
\dot{\tilde{Q}}=\tilde{Q} \sigma_{Q \times} \\
\dot{\tilde{R}}=\tilde{R} \Omega_{\times}-\Omega_{\times} \tilde{R}+\sigma_{R \times} \tilde{R} \\
\dot{\tilde{\xi}}=\left(-\Omega_{\times}+\phi_{\perp} I_{3}\right) \tilde{\bar{\xi}}+\sigma_{\bar{\xi}}
\end{array}\right.
$$

For analysis purposes let us assume that $\bar{\xi}, \Omega$ and $\phi_{\perp}$ remain bounded for all time, which is a completely reasonable assumption.

The following step involves developing first order approximations of the error system (17) and of the measurement equation (8). From the Rodrigues' formula, one deduces the following first order approximations of $\tilde{Q}$ and $\tilde{R}$

$$
\begin{aligned}
& \tilde{Q}=I+\lambda_{\tilde{Q} \times}+O\left(\left|\lambda_{\tilde{Q}}\right|^{2}\right) \\
& \tilde{R}=I+\lambda_{\tilde{R} \times}+O\left(\left|\lambda_{\tilde{R}}\right|^{2}\right)
\end{aligned}
$$

with $\lambda_{\tilde{Q}}, \lambda_{\tilde{R}} \in S^{2}$. One then deduces from the first two equations of (17) and (18) that in first order approximations

$$
\dot{\lambda}_{\tilde{Q}}=\sigma_{Q}+O\left(\left|\lambda_{\tilde{Q}}\right|\left|\sigma_{Q}\right|\right)
$$

and

$$
\begin{aligned}
\dot{\lambda}_{\tilde{R} \times} & =\lambda_{\tilde{R} \times} \Omega_{\times}-\Omega_{\times} \lambda_{\tilde{R} \times}+\sigma_{R \times}+O\left(\left|\lambda_{\tilde{R}}\right|^{2}\right)+O\left(\left|\lambda_{\tilde{R}}\right|\left|\sigma_{R}\right|\right) \\
& =\left(\lambda_{\tilde{R}} \times \Omega\right)_{\times}+\sigma_{R \times}+O\left(\left|\lambda_{\tilde{R}}\right|^{2}\right)+O\left(\left|\lambda_{\tilde{R}}\right|\left|\sigma_{R}\right|\right)
\end{aligned}
$$

which yields

$$
\dot{\lambda}_{\tilde{R}}=-\Omega_{\times} \lambda_{\tilde{R}}+\sigma_{R}+O\left(\left|\lambda_{\tilde{R}}\right|^{2}\right)+O\left(\left|\lambda_{\tilde{R}}\right|\left|\sigma_{R}\right|\right)
$$

As for the measurement equation (8), this homography expression can be developed in first order approximations as

$$
\begin{aligned}
& R^{\top} H-I_{3}=\bar{\xi} \eta^{\top}=\bar{\xi}\left(Q^{\top} e_{3}\right)^{\top} \\
\Rightarrow & \tilde{R}^{\top} \hat{R}^{\top} H-I_{3}=(\tilde{\bar{\xi}}+\hat{\bar{\xi}})\left(\hat{Q}^{\top} \tilde{Q}^{\top} e_{3}\right)^{\top} \\
\Rightarrow & \left(I_{3}-\lambda_{\tilde{R} \times}\right) \hat{R}^{\top} H-I_{3} \\
= & (\tilde{\bar{\xi}}+\hat{\bar{\xi}})\left(\hat{Q}^{\top}\left(I_{3}-\lambda_{\tilde{Q} \times}\right) e_{3}\right)^{\top}+O\left(\left|\lambda_{\tilde{Q}}\right|^{2}\right)+O\left(\left|\lambda_{\tilde{R}}\right|^{2}\right) \\
\Rightarrow & \left(\hat{R}^{\top} H-I_{3}\right)-\lambda_{\tilde{R} \times}\left(\hat{R}^{\top} H\right) \\
= & \hat{\bar{\xi}}\left(\hat{Q}^{\top} e_{3}\right)^{\top}+\tilde{\bar{\xi}}\left(\hat{Q}^{\top} e_{3}\right)^{\top}+\hat{\bar{\xi}}\left(\hat{Q}^{\top} e_{3 \times} \lambda_{\tilde{Q}}\right)^{\top} \\
& +O\left(\left|\lambda_{\tilde{Q}}\right|^{2}\right)+O\left(\left|\lambda_{\tilde{R}}\right|^{2}\right) \\
\Rightarrow & \left(\hat{R}^{\top} H-I_{3}\right)-\hat{\bar{\xi}}\left(\hat{Q}^{\top} e_{3}\right)^{\top} \\
= & \lambda_{\tilde{R} \times}\left(\hat{R}^{\top} H\right)+\tilde{\bar{\xi}}\left(\hat{Q}^{\top} e_{3}\right)^{\top}-\lambda_{\tilde{Q}, 2} \hat{\bar{\xi}}\left(\hat{Q}^{\top} e_{1}\right)^{\top}+\lambda_{\tilde{Q}, 1} \hat{\bar{\xi}}\left(\hat{Q}^{\top} e_{2}\right)^{\top} \\
& +O\left(\left|\lambda_{\tilde{Q}}\right|^{2}\right)+O\left(\left|\lambda_{\tilde{R}}\right|^{2}\right)
\end{aligned}
$$

Note that the last equality only involves the first two components of $\lambda_{\tilde{Q}}$ (i.e. $\lambda_{\tilde{Q}, 1}$ and $\lambda_{\tilde{Q}, 2}$ ) and can be equivalently written as

$Y:=\left[\begin{array}{c}\left(\hat{R}^{\top} H-I_{3}\right)\left(\hat{Q}^{\top} e_{3}\right)-\hat{\bar{\xi}} \\ \left(\hat{R}^{\top} H-I_{3}\right)\left(\hat{Q}^{\top} e_{2}\right) \\ \left(\hat{R}^{\top} H-I_{3}\right)\left(\hat{Q}^{\top} e_{1}\right)\end{array}\right]$
$=\left[\begin{array}{l}-\left(\left(\hat{R}^{\top} H\right)\left(\hat{Q}^{\top} e_{3}\right)\right)_{\times} \lambda_{\tilde{R}}+\hat{\bar{\xi}} \\ -\left(\left(\hat{R}^{\top} H\right)\left(\hat{Q}^{\top} e_{2}\right)\right)_{\times} \lambda_{\tilde{R}}+\lambda_{\tilde{Q}, 1} \hat{\bar{\xi}} \\ -\left(\left(\hat{R}^{\top} H\right)\left(\hat{Q}^{\top} e_{1}\right)\right)_{\times} \lambda_{\tilde{R}}-\lambda_{\tilde{Q}, 2} \hat{\bar{\xi}}\end{array}\right]+O\left(\left|\lambda_{\tilde{Q}}\right|^{2}\right)+O\left(\left|\lambda_{\tilde{R}}\right|^{2}\right)$ 
From (19), (20), the third equation of (17), and (21), one obtains in first order approximations the system in compact form (1) with output $Y$ defined in (21) and

$$
\left\{\begin{array}{c}
X:=\left[\begin{array}{c}
\lambda_{\tilde{Q}, 1} \\
\lambda_{\tilde{Q}, 2} \\
\lambda_{\tilde{R}}^{\tilde{\bar{\xi}}} \\
\overline{\bar{\xi}}
\end{array}\right], \quad U:=\left[\begin{array}{c}
\sigma_{Q, 1} \\
\sigma_{Q, 2} \\
\sigma_{R} \\
\sigma_{\bar{\xi}}
\end{array}\right] \\
A(t):=\left[\begin{array}{cccc}
0 & 0 & 0_{1 \times 3} & 0_{1 \times 3} \\
0 & 0 & 0_{1 \times 3} & 0_{1 \times 3} \\
0_{3 \times 1} & 0_{3 \times 1} & -\Omega_{\times} & 0_{3 \times 3} \\
0_{3 \times 1} & 0_{3 \times 1} & 0_{3 \times 3} & -\Omega_{\times}+\phi_{\perp} I_{3}
\end{array}\right] \\
C(X, t):=\left[\begin{array}{cccc}
0_{3 \times 1} & 0_{3 \times 1} & -\left(\left(\hat{R}^{\top} H\right)\left(\hat{Q}^{\top} e_{3}\right)\right)_{\times} & I_{3} \\
\hat{\bar{\xi}} & 0_{3 \times 1} & -\left(\left(\hat{R}^{\top} H\right)\left(\hat{Q}^{\top} e_{2}\right)\right)_{\times} & 0_{3 \times 3} \\
0_{3 \times 1} & -\overline{\bar{\xi}} & -\left(\left(\hat{R}^{\top} H\right)\left(\hat{Q}^{\top} e_{1}\right)\right)_{\times} & 0_{3 \times 3}
\end{array}\right]
\end{array}\right.
$$

The fact that $X=\left[X_{1}^{\top}, X_{2}^{\top}\right]^{\top}$ with $X_{1}:=$ $\left[\lambda_{\tilde{Q}, 1}, \lambda_{\tilde{Q}, 2}, \lambda_{\tilde{R}}^{\top}\right]^{\top} \in \mathcal{B}_{2}^{5}$ (the closed ball in $\mathbb{R}^{5}$ of radius equal to 2$), X_{2}:=\bar{\xi} \in \mathbb{R}^{3}$, together with the particular form of the matrix $A$ as (2), allows one to obtain the expression of the innovation terms from the input $U$ calculated according to (3) and (4) where the matrices $D$ and $S$ (involved in (4)) are chosen larger than some constant positive matrix.

\section{B. Observability and stability analysis}

According to [2] the equilibrium $X=0$ is locally exponentially stable, provided that the pair $\left(A^{\star}(t), C^{\star}(t)\right)$ with $A^{\star}(t)=A(t)$ and $C^{\star}(t):=C(0, t)$ is uniformly observable. By setting $X=0$ and looking at (22) one obtains

$$
\begin{aligned}
C^{\star} & =\left[\begin{array}{cccc}
0_{3 \times 1} & 0_{3 \times 1} & -\left(\left(I_{3}+\bar{\xi} \eta^{\top}\right)\left(Q^{\star \top} e_{3}\right)\right)_{\times} & I_{3} \\
\bar{\xi} & 0_{3 \times 1} & -\left(\left(I_{3}+\bar{\xi} \eta^{\top}\right)\left(Q^{\star \top} e_{2}\right)\right)_{\times} & 0_{3 \times 3} \\
0_{3 \times 1} & -\bar{\xi} & -\left(\left(I_{3}+\bar{\xi} \eta^{\top}\right)\left(Q^{\star \top} e_{1}\right)\right)_{\times} & 0_{3 \times 3}
\end{array}\right] \\
= & {\left[\begin{array}{cccc}
0_{3 \times 1} & 0_{3 \times 1} & -(\eta+\bar{\xi})_{\times} & I_{3} \\
\bar{\xi} & 0_{3 \times 1} & -\left(Q^{\star \top} e_{2}\right)_{\times} & 0_{3 \times 3} \\
0_{3 \times 1} & -\bar{\xi} & -\left(Q^{\star \top} e_{1}\right)_{\times} & 0_{3 \times 3}
\end{array}\right] }
\end{aligned}
$$

with $Q^{\star} \in S O(3)$ satisfying $Q^{\star \top} e_{3}=\eta$ and $\dot{Q}^{\star}=Q^{\star} \Omega_{\times}$

For later use, let $q_{i}^{\star} \in S^{2}, i=1,2,3$, denote the $i$-th row of $Q^{\star}$ (i.e. $q_{i}^{\star}=Q^{\star \top} e_{i}$ ).

Theorem 1 Assume that there exists a positive number $\nu$ such that $\forall t>0$

$$
\frac{1}{\delta} \int_{t}^{t+\delta}|\bar{\xi}(\tau) \times \eta(\tau)| \mathrm{d} \tau \geq \nu
$$

Assume also that $\Omega, \phi, \bar{\xi}$ remain uniformly bounded. Then, the pair $\left(A^{*}, C^{*}\right)$ is uniformly observable. Assume that the matrices $D$ and $S$ involved in (4) are chosen larger than some constant positive matrix. Then, the equilibrium $\left(\tilde{Q} e_{3}, \tilde{R}, \tilde{\xi}\right)=\left(e_{3}, I_{3}, 0\right)$ of the error system is locally exponentially stable.

Proof: According to Lemma 1 by choosing $M=C^{\star}$, then the pair $\left(A^{*}, C^{*}\right)$ is uniformly observable if $\exists \delta, \mu>0$ such that

$$
\frac{1}{\delta} \int_{t}^{t+\delta} \operatorname{det}\left(C^{\star \top}(\tau) C^{\star}(\tau)\right) \mathrm{d} \tau \geq \mu, \forall t>0
$$

We show thereafter that condition (23) is sufficient to guarantee (24). One verifies that

$$
C^{\star \top} C^{\star}=\left[\begin{array}{cccc}
|\bar{\xi}|^{2} & 0 & -\bar{\xi} q_{2 \times}^{\star} & 0_{1 \times 3} \\
0 & |\bar{\xi}|^{2} & \bar{\xi} q_{1 \times}^{\star} & 0_{1 \times 3} \\
q_{2 \times}^{\star} \bar{\xi} & -q_{1 \times}^{\star} \bar{\xi} & -(\eta+\bar{\xi})_{\times}^{2}-q_{1 \times}^{\star 2}-q_{2 \times}^{\star 2} & (\eta+\bar{\xi})_{\times} \\
0_{3 \times 1} & 0_{3 \times 1} & -(\eta+\bar{\xi})_{\times} & I_{3}
\end{array}\right]
$$

which can be rewritten as

$$
C^{\star \top} C^{\star}=|\bar{\xi}|^{2}\left[\begin{array}{cc}
I_{2} & \frac{1}{|\xi|^{2}} B \\
\frac{1}{|\xi|^{2}} B^{\top} & \frac{1}{|\xi|^{2}} G
\end{array}\right]
$$

where the expressions of $B \in \mathbb{R}^{2 \times 6}$ and $G \in \mathbb{R}^{6 \times 6}$ can be easily deduced. Thus, one deduces

$$
\begin{aligned}
& \operatorname{det}\left(C^{\star \top} C^{\star}\right)=|\bar{\xi}|^{4} \operatorname{det}\left(G-\frac{1}{|\bar{\xi}|^{2}} B^{\top} B\right) \\
& =|\bar{\xi}|^{4} \operatorname{det}\left[\begin{array}{c}
-(\eta+\bar{\xi})_{\times}^{2}-q_{1 \times}^{\star} \pi_{\frac{\bar{\xi}}{|\xi|}} q_{1 \times}^{\star}-q_{2 \times}^{\star} \pi_{\frac{\bar{\xi}}{|\bar{\xi}|}} q_{2 \times}^{\star} \\
-(\eta+\bar{\xi})_{\times}
\end{array}\left(\begin{array}{c}
\eta_{3} \\
I
\end{array}\right]\right. \\
& =|\bar{\xi}|^{4} \operatorname{det}\left(-q_{1 \times}^{\star} \pi_{\left.\frac{\bar{\xi}}{|\bar{\xi}|} q_{1 \times}^{\star}-q_{2 \times}^{\star} \pi_{\frac{\bar{\xi}}{|\xi|}} q_{2 \times}^{\star}\right)}\right. \\
& =|\bar{\xi}|^{4} \operatorname{det}\left(Q^{\star \top}\left(-e_{1 \times} \pi_{\frac{Q^{\star} \bar{\xi}}{|\xi|}} e_{1 \times}-e_{2 \times} \pi_{\frac{Q^{\star} \bar{\xi}}{|\xi|}} e_{2 \times}\right) Q^{\star}\right) \\
& =|\bar{\xi}|^{4} \operatorname{det}\left(-e_{1 \times} \pi_{\eta_{\bar{\xi}}^{\star}} e_{1 \times}-e_{2 \times} \pi_{\eta_{\bar{\xi}}^{\star}} e_{2 \times}\right) \\
& =|\bar{\xi}|^{4} \operatorname{det}\left(e_{3} e_{3}^{\top}+\eta_{\bar{\xi}}^{\star} \eta_{\bar{\xi}}^{\star}+\left(\eta_{\bar{\xi}}^{\star} \times e_{3}\right)\left(\eta_{\bar{\xi}}^{\star} \times e_{3}\right)^{\top}\right) \\
& =|\bar{\xi}|^{4}\left|\eta_{\bar{\xi}}^{\star} \times e_{3}\right|^{4} \\
& =|\bar{\xi} \times \eta|^{4}
\end{aligned}
$$

with $\eta_{\bar{\xi}}^{\star}:=\frac{Q^{\star} \bar{\xi}}{|\bar{\xi}|}$. From the Cauchy-Schwarz integral inequality, one deduces (using (23))

$$
\begin{aligned}
& \int_{t}^{t+\delta}|\bar{\xi}(\tau) \times \eta(\tau)|^{4} \mathrm{~d} \tau \geq \frac{1}{\delta}\left(\int_{t}^{t+\delta}|\bar{\xi}(\tau) \times \eta(\tau)|^{2} \mathrm{~d} \tau\right)^{2} \\
& \geq \frac{1}{\delta^{3}}\left(\int_{t}^{t+\delta}|\bar{\xi}(\tau) \times \eta(\tau)| \mathrm{d} \tau\right)^{4} \geq \delta \nu^{4}
\end{aligned}
$$

One finally deduces (24) with $\mu=\nu^{4}$. The remainder of the proof then directly follows by application of Theorem 3.1 and Corollary 3.2 in [2].

\section{Simulation Results}

In this section the robustness and performance of the proposed observer is demonstrated through simulation results by considering two scenarios in particular. For the simulations, we consider that a monocular camera is attached to an aerial drone performing some specific trajectories and observing a planar target. In the first scenario, the drone realizes an aggressive periodical trajectory and noise is introduced to the measurements used in the proposed observer, namely $H$, $\phi, \phi_{\perp}$ and $\Omega$. While in the second scenario, we consider the case where the camera trajectory passes through the reference position (i.e. the camera translation vanishes) again with the introduction of noise in the measurements.

With Matlab Simulink, we try to simulate the image noise by introducing white Gaussian noise of the level of about 10 percent of the real values on each individual component of the homography matrix $H$. We also introduce white 

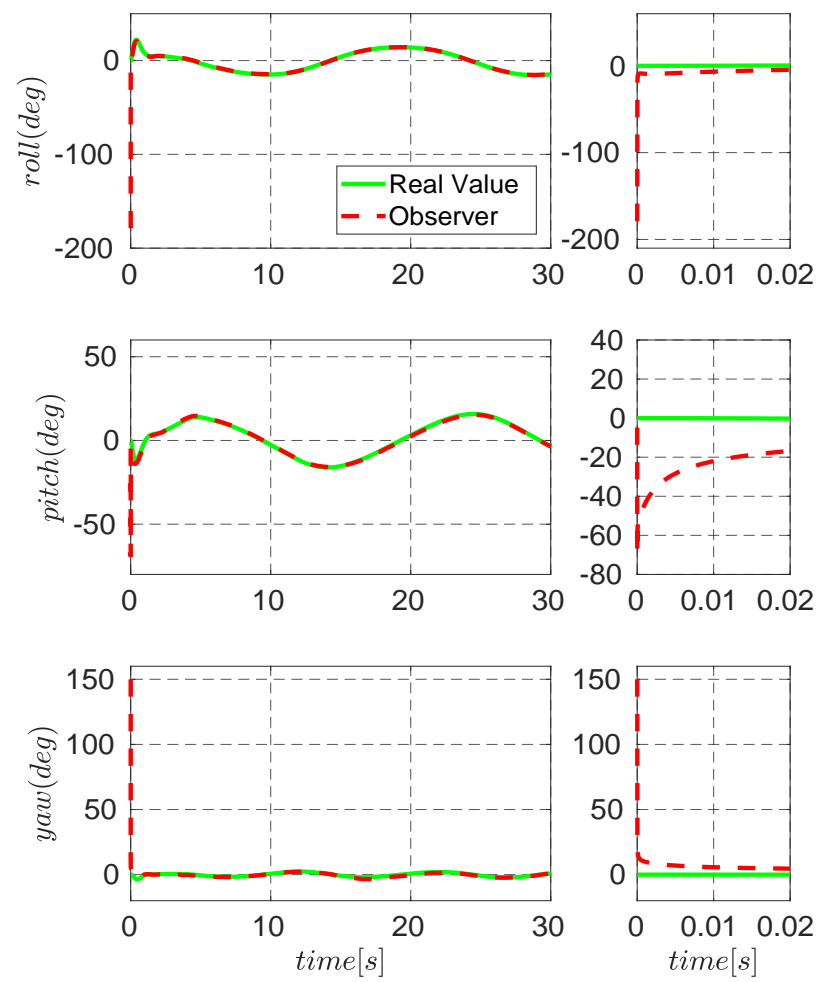

Fig. 2. Scenario 1 - Estimated and real attitudes represented by roll, pitch and yaw Euler angles $(\mathrm{deg})$ versus time $(s)$ (LEFT) and zooms (RIGHT)
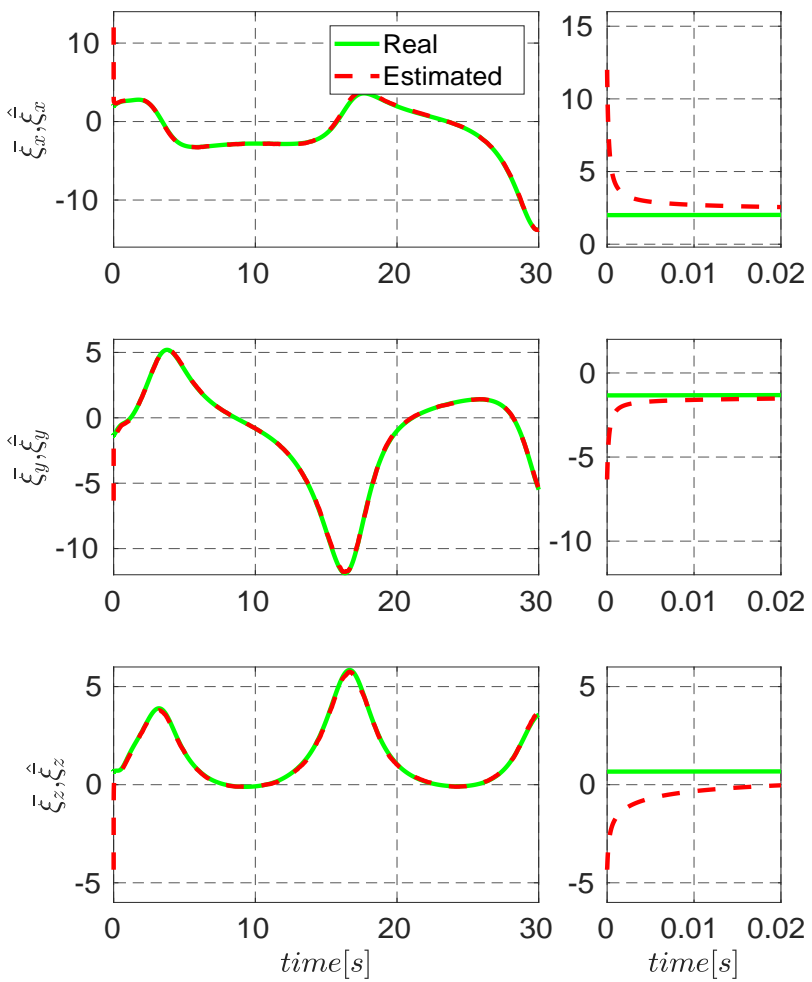

Fig. 3. Scenario 1 - Estimated and real scaled position versus time(s) (LEFT) and zooms (RIGHT)

Gaussian noise of variance of $1(\mathrm{deg} / \mathrm{s}), 0.1$ and 0.1 on the measurements of $\Omega, \phi$ and $\phi_{\perp}$, respectively. In both simulated scenarios, the matrices $S$ and $D^{-1}$ involved in the CRE (4) are interpreted as covariance matrices of the additive noise on the system state and output respectively, and the

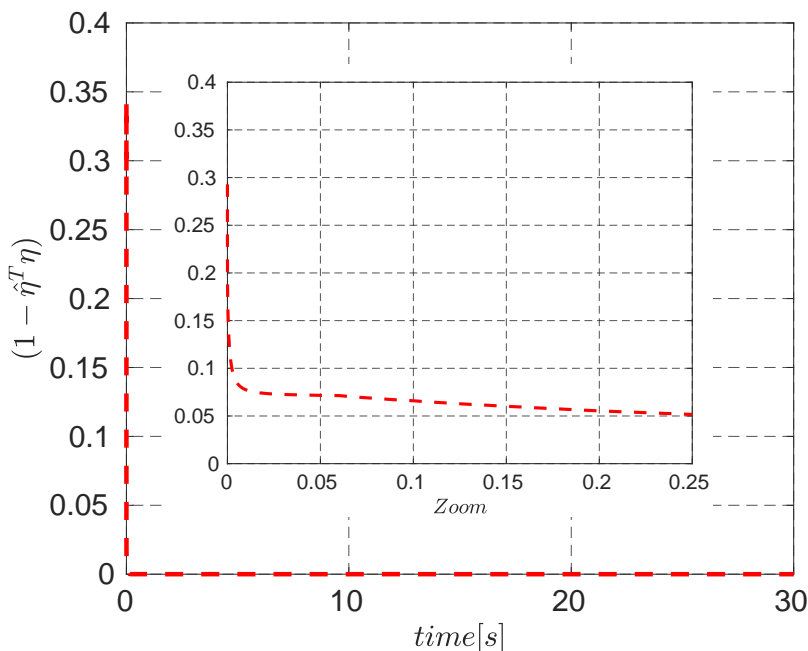

Fig. 4. Scenario 1 - Estimation error of the normal vector estimate represented by $1-\hat{\eta}^{T} \eta$

observer is tuned in a similar way like Kalman-Bucy filters. Thus, the following parameters are chosen: $P(0)=50 I_{9}$, $D=100 I_{9}, S=\operatorname{diag}\left(0.0175^{2} I_{2} ; 0.0175^{2} I_{3} ; 0.1^{2} I_{3}\right)$.

The following initial estimation errors are considered for both scenarios: $\tilde{\bar{\xi}}(0)=[10,-5,5]^{\top}$, quat $_{\tilde{R}}(0)=[0.0436,0.2586,0.965,0]^{\top}$ (corresponding to errors in roll, pitch and yaw Euler angles of $178.7(\mathrm{deg}), \quad-4.8(\mathrm{deg}), \quad-150(\mathrm{deg}), \quad$ respectively $)$, quat $_{\hat{Q}}(0)=[0.9239,0.3827,0,0]^{\top}$ (corresponding to an angle error of $45(\mathrm{deg})$ between $\eta(0)$ and $\hat{\eta}(0))$, where the scene is chosen such that $i=e_{3}$ and $\stackrel{\circ}{d}=3(\mathrm{~m})$.

Scenario 1: For this particular case the drone is commanded to perform a complex periodical trajectory in the inertial frame given by

$\xi=[10 \cos (t / \sqrt{10})-4,10 \sin (t / \sqrt{10})-4,2 \sin (0.3 \pi t / 2)+2]^{\top}$

The time evolutions of the estimated and real attitudes (represented by Euler angles), the attitude error estimate (represented by trace $(I-\tilde{R})$ ) as well as the scaled position error estimate and the estimation error of the normal vector to the planar scene (represented by $1-\hat{\eta}^{\top} \eta$ ) are shown in Figs $2-4$, respectively. From these figures, it can be clearly seen that all the estimated variables converge to the real ones after a short transition period of a few miliseconds despite the large initial estimation errors. The above results also show that the proposed observer is robust to noisy measurements as the latter marginally affects the overall performance of the proposed observer.

Scenario 2: In this case the drone performs a rectilinear sinusoidal trajectory that passes through the reference position (i.e. the camera translation vanishes) with added noise on the measurements of $H, \phi, \phi_{\perp}$, and $\Omega$ as in the previous simulation. The reference trajectory in the inertial frame is given by $\xi=[5 \sin (\pi t / 3), 0,0]^{\top}(m)$. It is worth noting that in such a degenerate case (i.e. when the camera translation vanishes) all traditional algebraic approaches fail to obtain a correct estimate of the normal vector (there exists in fact an infinity of solutions) whereas our approach still works since the uniform observability condition (23) is always satisfied. 
This is also because of the fact that the state estimates are propagated over time. From Figs. 5-7 it can be clearly seen that for this degenerate case and even with the addition of noise to the system measurements, the proposed observer is robust enough to provide a convincing performance in terms of convergence rate, smooth transient phase, and filtering of measurement noise.
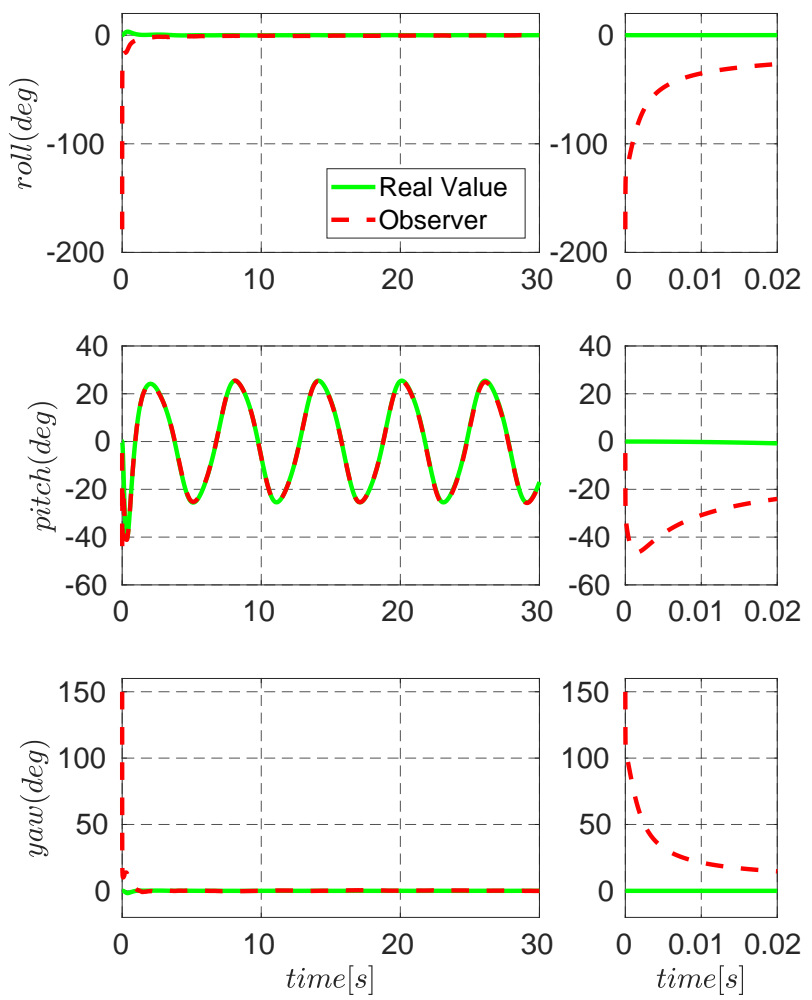

Fig. 5. Scenario 2 - Estimated and real attitudes represented by roll, pitch and yaw Euler angles (deg) versus time ( $s$ (LEFT) and zooms (RIGHT)

\section{CONCLUSIONS}

In this paper a novel approach for solving the classical problem of homography decomposition has been proposed by designing a nonlinear Riccati observer. To our knowledge this is the first study to address the homography decomposition problem by exploiting the system dynamics. By exploiting the temporal correlation of image sequences and the natural low-pass response of the observer, the resulting estimates are robust and less prone to measurement noise. Exhaustive observability and stability analysis has been carried out in order to support the proposed observer. At the end convincing simulation results have been presented to show the performance of the observer.

Acknowledgment: This work was supported by the ANR Astrid CONGRE (ANR-18-ASTR-0006), FUI GreenExplorer and ANR EQUIPEX Robotex projects.

\section{REFERENCES}

[1] O. D. Faugeras and F. Lustman. Motion and structure from motion in a piecewise planar environment. International Journal of Pattern Recognition and Artificial Intelligence, 2(03):485-508, 1988.

[2] T. Hamel and C. Samson. Riccati observers for the nonstationary PnP problem. volume 63, pages 726-741, 2017.
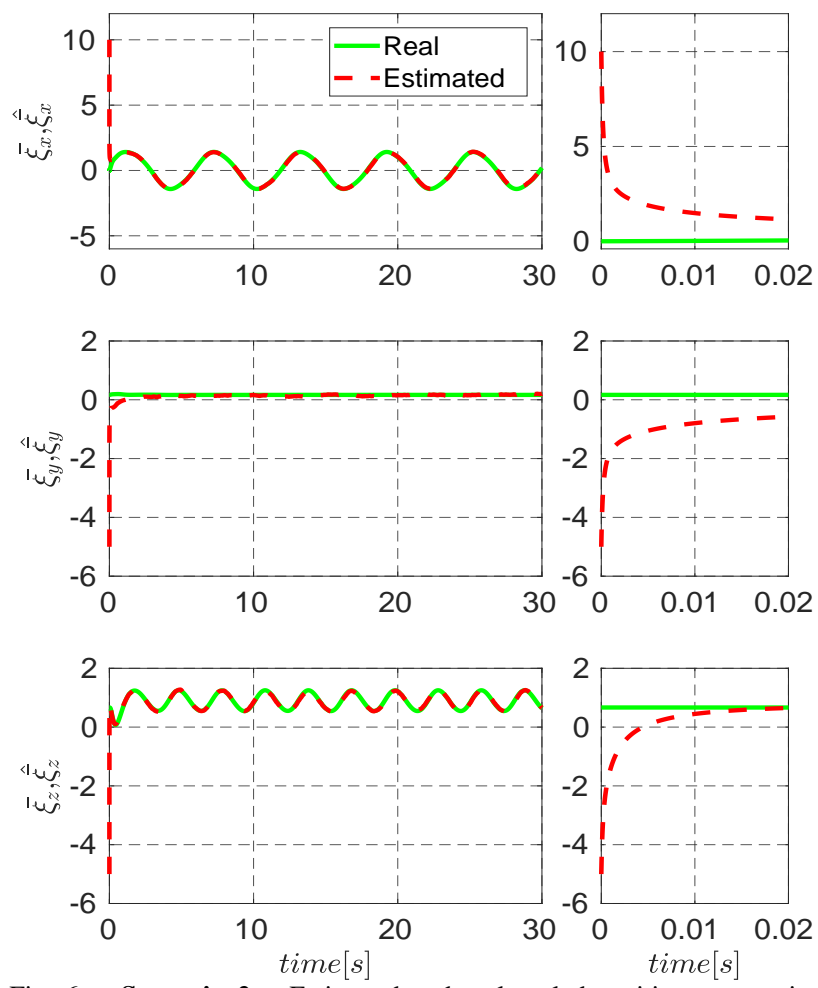

Fig. 6. Scenario 2 - Estimated and real scaled position versus time(s) (LEFT) and zooms (RIGHT)

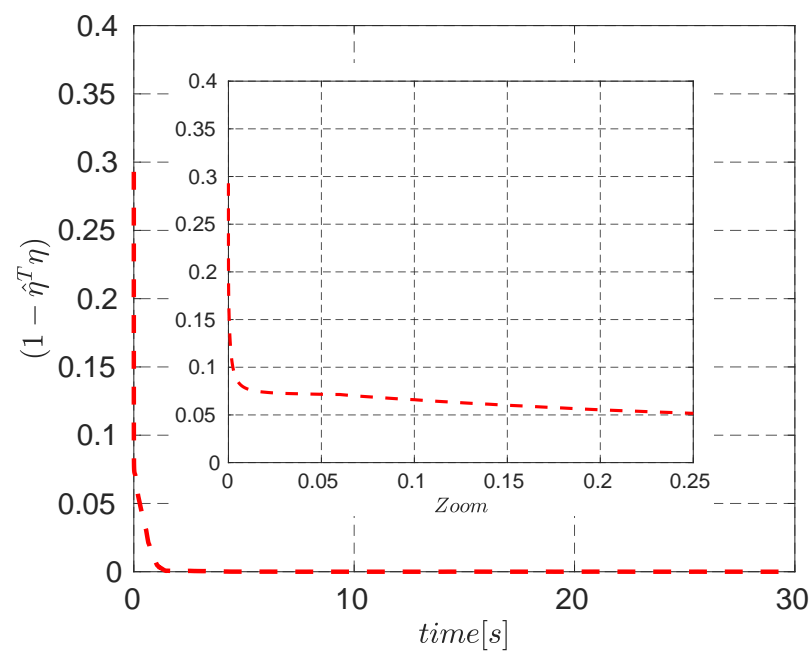

Fig. 7. Scenario 2 - Estimation error of the normal vector estimate represented by $1-\hat{\eta}^{T} \eta$

[3] R. Hartley and A. Zisserman. Multiple view geometry in computer vision. Cambridge university press, 2003.

[4] M.-D. Hua and G. Allibert. Riccati observer design for pose, linear velocity and gravity direction estimation using landmark position and IMU measurements. In IEEE Conference on Control Technology and Applications (CCTA), pages 1313-1318, 2018.

[5] E. Malis, F. Chaumette, and S. Boudet. 2 1/2 D visual servoing. IEEE Transactions on Robotics and Automation, 15(2):238-250, 1999.

[6] E. Malis and M. Vargas. Deeper understanding of the homography decomposition for vision-based control. 2007.

[7] N. Manerikar, M.-D. Hua, and T. Hamel. Homography observer design on special linear group SL(3) with application to optical flow estimation. In European Control Conference (ECC), pages 1-5, 2018.

[8] G. G. Scandaroli. Visuo-inertial data fusion for pose estimation and self-calibration. PhD thesis, Université Nice Sophia Antipolis, 2013.

[9] Z. Zhang and A. R. Hanson. 3D reconstruction based on homography mapping. Proc. ARPA96, pages 1007-1012, 1996. 